\title{
Severe kyphoscoliosis causing marked displacement and rotation of right kidney with acute kinking of right ureter resulting in hydronephrosis
}

Spinal Cord (2005) 43, 195-196. doi:10.1038/sj.sc.3101696; Published online 23 November 2004

\begin{abstract}
Although adverse effects of severe kyphoscoliosis upon thoracic organs have been well publicised, the impact of severe spinal deformity upon the kidneys has not received attention. We report a patient with spina bifida and severe kyphoscoliosis, in whom kyphoscoliotic deformity resulted in marked displacement and rotation of the right kidney with acute kinking of the right ureter at the pelviureteric junction, thus causing hydronephrosis.
\end{abstract}

\section{Case report}

A male patient with spina bifida had been managing the bladder by wearing a urostomy bag around the penis. Intravenous urography (IVU), performed when he was 19 years old (1996), showed normal kidneys. This patient developed progressively worsening kyphoscoliosis. Due to spinal curvature, he exhibited abdominal bulge on the left side; the sacrum was deviated towards his left side and he developed a large pressure sore over the sacrum. In September 2002, ultrasound scan, performed routinely, revealed moderate right hydronephrosis. He had no symptoms related to the urinary tract. In February 2003, IVU showed a normal left kidney; there was delay in excretion of contrast by the hydronephrotic right kidney. Hydronephrosis was presumed to be due to unsatisfactory drainage of the neuropathic bladder. The patient felt that intermittent catheterisation would not suit his lifestyle. The flange of the urostomy bag was digging into the suprapubic region. Therefore, indwelling catheter drainage was established in February 2003 and he was prescribed modified-release formulation of oxybutynin $10 \mathrm{mg}$ a day.

X-ray of abdomen revealed severe degree of spinal curvature (Figure 1). No opaque calculus was seen in the kidneys, ureters or urinary bladder. In April 2003, CT of abdomen was performed to look for urinary calculi, which might not be visible in a plain film because of spinal deformity. CT scan demonstrated marked medial and downward displacement of the right kidney because of severe kyphoscoliosis. The kidney was situated at the level of $\mathrm{L}-4 / 5$. The renal pelvis was rotated posterolaterally. There was no regression of hydronephrosis despite indwelling catheter drainage and oral oxybutynin therapy. There was no calculus impacted at the pelviureteric junction. The ureter below the level of the pelviureteric junction on the right was of normal calibre.

CT angiogram demonstrated the kidneys and aorta to be rotated so much that the axial scan of the abdomen gave a view simulating coronal section of kidneys and major blood vessels. The bony pelvis was rotated. The left kidney was located laterally above the left ilium. The right renal pelvis was distended (Figure 2). The right ureter maintained its normal relationship to the vasculature, in as much as it was lying anterior to the common iliac artery and vein, which suggested that

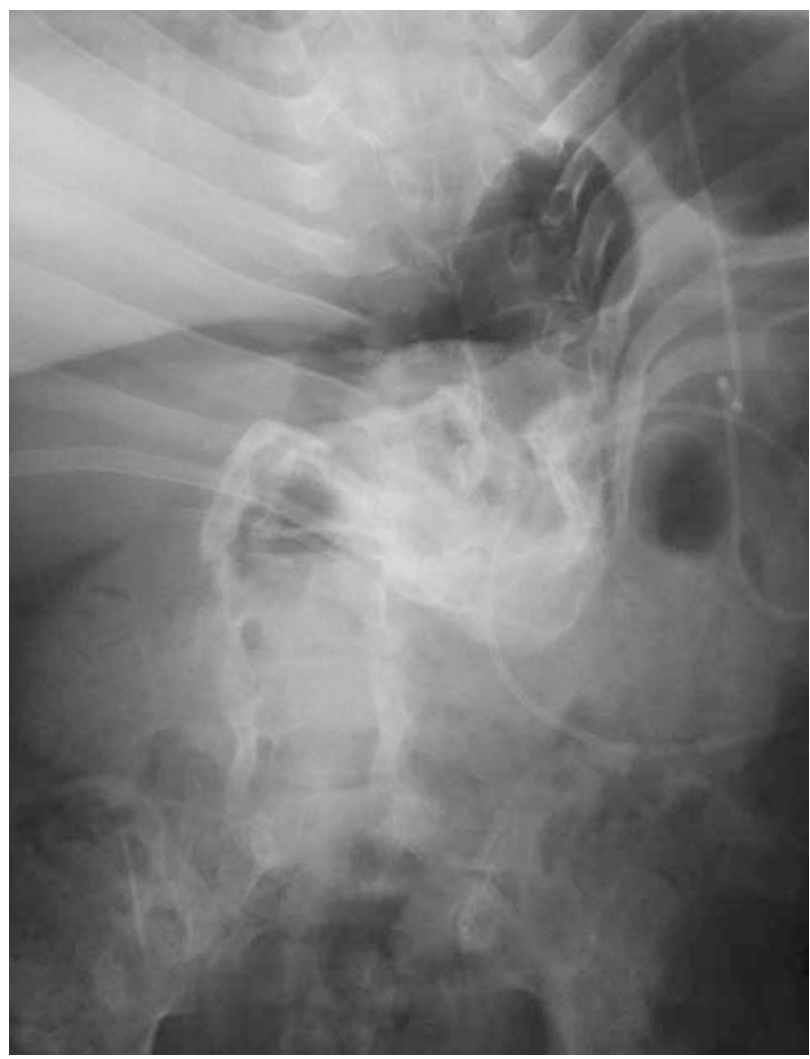

Figure 1 X-ray of abdomen (14 May 2003) shows severe kyphoscoliosis. The space between the last rib and iliac crest is reduced because of spinal curvature. No opaque calculus was seen in the kidneys, ureters or urinary bladder 


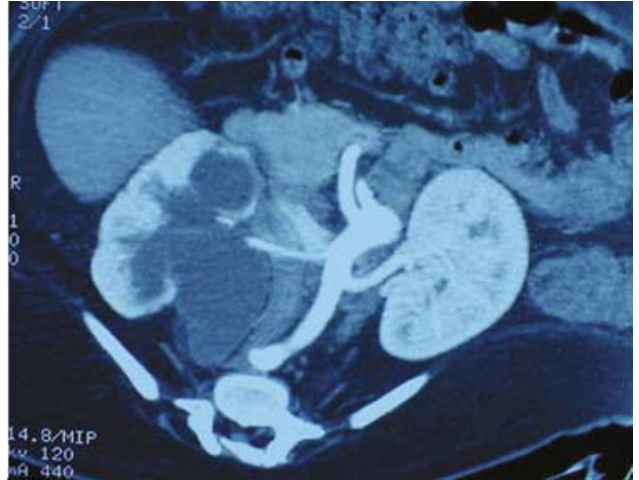

Figure 2 CT angiography (11 June 2003) shows the aorta and both renal arteries. The bony pelvis is rotated. The left kidney is located laterally above the left ilium. The right kidney is hydronephrotic. The kidneys and aorta are rotated so much that the axial scan of abdomen gives a view simulating coronal section of the kidneys and major blood vessels

hydronephrosis was due to the kinking of the ureter at the pelviureteic junction as a result of the patient's bodily habitus (severe kyphoscoliotic deformity and pelvic obliquity) rather than it being anything to due to the relationship between the ureter and the iliac vessels. Oblique coronal 3-D reconstruction using Voxar 3D software (courtesy of Voxar Limited, UK, www.voxar.com) confirmed acute kinking of the right ureter at the ureteropelvic junction (Figure 3).

Pulmonary function tests revealed evidence of restrictive lung disease. MAG-3 renogram showed a normal left renogram curve. Right renogram showed slow excretion. Relative function of the left kidney was $91 \%$ and relative function of the right kidney was only $9 \%$. After discussing risks and benefits of surgery with the patient and his mother, an informed decision was taken against surgical correction for kinking of the right ureter.

In conclusion, this patient, who had spina bifida, developed marked kyphoscoliosis. Severe degree of

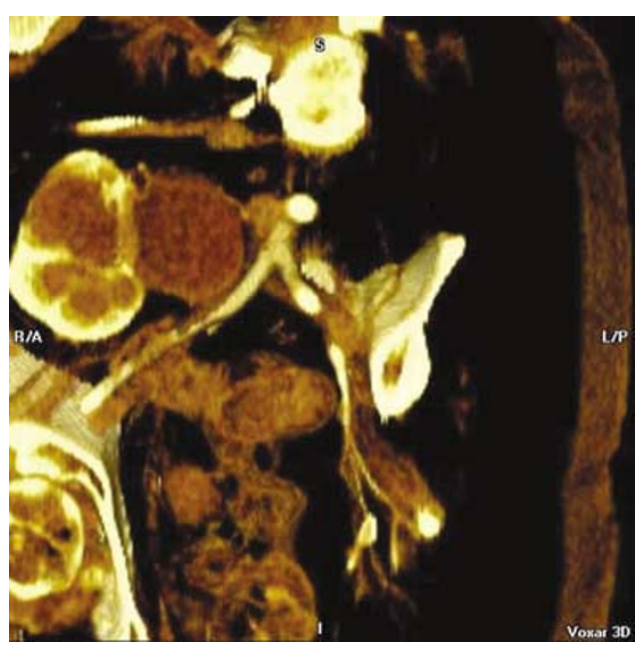

Figure 3 3-D reconstruction of CT angiogram using Voxar 3D software: oblique $20 \mathrm{~mm}$ coronal slab shows pelvic obliquity. The right kidney is displaced inferiorly and rotated as well. The right renal pelvis is distended. There is acute kinking of the right ureter at the ureteropelvic junction

kyphoscoliosis, in addition to causing restrictive lung disease, resulted in displacement and rotation of the right kidney, which led to acute kinking of the right ureter at the pelviureteric junction, thus causing hydronephrosis.

\section{Acknowledgements}

The authors thank Mrs Sheila Thomas, Astra Tech Ltd, Brunel Way, Stonehouse, Glos, GL10 3SX for sponsoring the printing of colour illustrations.

$$
\begin{array}{r}
\text { S Vaidyanathan }{ }^{1}, \mathrm{PL} \text { Hughes }^{1}, \mathrm{G} \mathrm{Singh}{ }^{1}, \mathrm{BM} \text { Soni }{ }^{1} \\
\text { and JWH Watt }
\end{array}
$$

